\title{
Reliability of a nutrition screening tool for use in cancer patients
}

\author{
S. M. Lewis ${ }^{1}$, H. L. Edwards-Jones ${ }^{1}$ and F. Minto ${ }^{2}$ \\ ${ }^{1}$ Velindre Cancer Centre, Cardiff CF14 2TL, UK and ${ }^{2}$ University of Wales Institute Cardiff, Cardiff CF5 2YB, UK
}

Prevalence of malnutrition within the oncology setting has been well documented ${ }^{(1)}$. Nutrition screening has been identified as best practice to identify patients at risk of malnutrition ${ }^{(2)}$. The Malnutrition Universal Screening Tool (MUST) has been widely accepted as the tool of choice yet it has not been validated for use in the oncology setting. Within a regional cancer centre a local tool, the Moreland Score, has been adopted as the mandatory screening tool since the mid-1990s. It consists of a scoring system addressing issues such as weight loss, appetite and nutritional symptoms related to oncological treatment. Validity and reliability of the tool remain to be tested. Previous work indicates significant correlation with the PG-SGA, a validated tool in the oncology setting ${ }^{(3)}$.

The aim of the study was to investigate the inter-rater reliability of the local nutrition screening tool and identify potential areas for change of practice.

A prospective nutrition screening survey was undertaken between July 2009 and December 2009. Hundred patients were routinely screened on admission to the cancer centre by nursing staff; $55 \%$ men, $45 \%$ females; $30 \%$ upper gastrointestinal (GI) cancer; $13 \%$ head and neck cancer, $13 \%$ lower GI tract and $44 \%$ various tumour sites. Inter-rater reliability was assessed using two trained researchers. Researcher 1 completed nutrition screening for all 100 patients and researcher 2 completed for 20 patients. All screening took place on the same day at different times and researchers were blinded to the results obtained. Kappa coefficients were calculated for agreement between nursing staff and the researchers. Prevalence of malnutrition for all admissions was $32 \%$ by nursing staff (Table 1). In contrast, both researchers identified a very high percentage of patients at risk (Table 1).

\begin{tabular}{lccc}
\hline Raters & $\%$ patients at risk $(n$ 100) & $\%$ patients not at risk $(n$ 100) & $\%$ patients at risk $(n$ 20) \\
\hline Nursing staff & 32 & 68 & 15 \\
Researcher 1 & 64 & 36 & 70 \\
Researcher 2 & N/A & N/A & 30 \\
\hline
\end{tabular}

Evaluation of the inter-rater reliability showed fair agreement between nursing staff with researcher 1, and nursing staff with both researchers (kappa value 0.24 and 0.27 respectively). In comparison, there was substantial agreement between the two trained researchers (kappa value 0.78).

The low level of agreement and lack of recognition could be due to the poor uptake of nutrition education by nursing staff. This study has identified that reliability of the tool is greater when specific training on nutrition screening is provided as shown by the two trained researchers. It is imperative that to improve the reliability of the tool a comprehensive nutrition training programme for nursing staff must be developed and implemented as mandatory.

1. Stratton RJ, Green CJ \& Elia M (2003) Disease-Related Malnutrition: An Evidence-Based Approach to Treatment. Oxon: CABI Publishing.

2. National Collaborating Centre for Acute Care (2006) http://www.evidence.nhs.uk/details.aspx?ci=http\%3a $\% 2$ f $\% 2$ fwww.nice.org.uk\% 2 fnicemedia\% 2flive \%2f10978\%2f29981\%2f29981.pdf\&hn=0\&rr=13944\&bv=10450

3. Lewis SM (2010) Comparison of three nutrition screening tools for patients with cancer in a regional cancer centre. Proc Nutr Soc 69(OCE2), E161. 